\title{
Luteinizing hormone inhibits cisplatin-induced apoptosis in human epithelial ovarian cancer cells
}

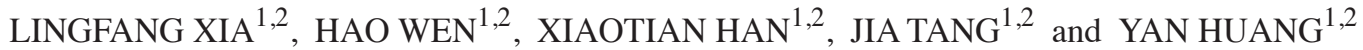 \\ ${ }^{1}$ Department of Gynecologic Oncology, Fudan University Shanghai Cancer Center; ${ }^{2}$ Department of Oncology, \\ Shanghai Medical College, Fudan University, Shanghai 200032, P.R. China
}

Received March 14, 2015; Accepted December 10, 2015

DOI: $10.3892 / \mathrm{ol} .2016 .4122$

\begin{abstract}
The elevation of Luteinizing hormone (LH) is commonly observed in epithelial ovarian cancer. This correlation suggests a causal relationship between LH and ovarian cancer. LH has been reported to inhibit apoptosis in ovarian cancer cells. Programmed cell death gene 6 (PDCD6), also known as apoptosis-linked gene-2, is an apoptotic mediator that is required for apoptosis to numerous death stimuli. Therefore, the aim of the present study was to determine whether PDCD6 may be induced by LH in ovarian cancer, and whether LH may affect the apoptosis through PDCD6. Flow cytometry was used to detect the effects of cisplatin on the induction of apoptosis by LH. PDCD6 expression was monitored by quantitative polymerase chain reaction and western blotting. The signaling transduction pathways were also investigated by western blotting. The present study demonstrated that LH reduced cisplatin-induced apoptosis in ovarian OVCAR-3 and SKOV-3 cancer cells. The results indicated that PDCD6 expression was inhibited by $\mathrm{LH}$. In addition, the inhibition of PDCD6, induced by LH, was mediated through the activation of the phosphatidylinositol 3-kinase/protein kinase B and p44/42 mitogen-activated protein kinase transduction signaling pathways. The present results suggest that $\mathrm{LH}$ affects the sensitivity of ovarian cancer cells to chemotherapy, primarily by signaling to inhibit apoptosis and to additionally suppress PDCD6.
\end{abstract}

\section{Introduction}

Ovarian cancer ranks fifth overall for cancer-associated mortalities in women, with lung, breast, colorectal and pancreatic cancer ranking 1-4, respectively, and accounts for 5\% of

Correspondence to: Dr Yan Huang, Department of Gynecologic Oncology, Fudan University Shanghai Cancer Center, 270 Dongan Road, Shanghai 200032, P.R. China

E-mail: huangyan19790315@163.com

Key words: luteinizing hormone, cisplatin, ovarian neoplasms, apoptosis, signal transduction pathways all cancer-associated mortalities in women (1). The overall five-year survival rate is $\sim 44 \%$, and only $27 \%$ for patients that are at the distant stage of the disease at diagnosis (1). The exact cause of epithelial ovarian cancer has not yet been determined, although follicle-stimulating hormone (FSH) and luteinizing hormone (LH) are understood to be associated with ovarian malignancy (2). With apoptosis and apoptosis-linked genes demonstrating key roles in ovarian cancer tumorigenesis $(3,4)$, it is important to note that FSH has been previously reported to inhibit apoptosis in ovarian cancer cells (5). However, little is currently understood regarding the association between $\mathrm{LH}$ and apoptosis in ovarian cancer, with further investigation required.

Programmed cell death gene 6 (PDCD6), also known as apoptosis-linked gene-2, is an apoptotic mediator that is required for apoptosis to occur in response to numerous death stimuli (6). It has recently been suggested that PDCD6 is an independent predictor of progression-free survival in patients with epithelial ovarian cancer (7). However, its effect on ovarian cancer apoptosis is currently unknown.

To understand the function and regulation of LH on ovarian cancer apoptosis, the present study investigated the effect of LH on apoptosis in vitro using flow cytometry. The protein and mRNA expression levels of PDCD6 were analyzed using western blotting and quantitative polymerase chain reaction (qPCR). The signal transduction pathways were also examined using western blotting.

\section{Materials and methods}

Reagents and antibodies. LH, SP600125 [a stress-activated protein kinase/c-Jun $\mathrm{NH}_{2}$-terminal kinase (SAPK/JNK) inhibitor], SB203580 [a p38 mitogen-activated protein kinase (MAPK) inhibitor], LY294002 [a phosphatidylinositol 3-kinase (PI3K) inhibitor] and U0126 [a p44/42 MAPK (extracellular signal-regulated kinase $1 / 2$; Erk1/2) inhibitor] were acquired from Sigma-Aldrich (St. Louis, MO, USA). The PDCD6 rabbit anti-human polyclonal antibody (cat. no. 12303-1-AP; dilution, 1:1,000) was purchased from Proteintech Group, Inc. (Chicago, IL, USA) for western blotting. The antibodies against phospho-protein kinase B (pAKT; rabbit anti-human monoclonal antibody; cat. no. 4060; dilution, 1:1,000), protein kinase B (AKT; rabbit anti-human monoclonal antibody; cat. no. 9272; dilution, 1:1,000), phospho-c-Jun (pJUN; rabbit 
A

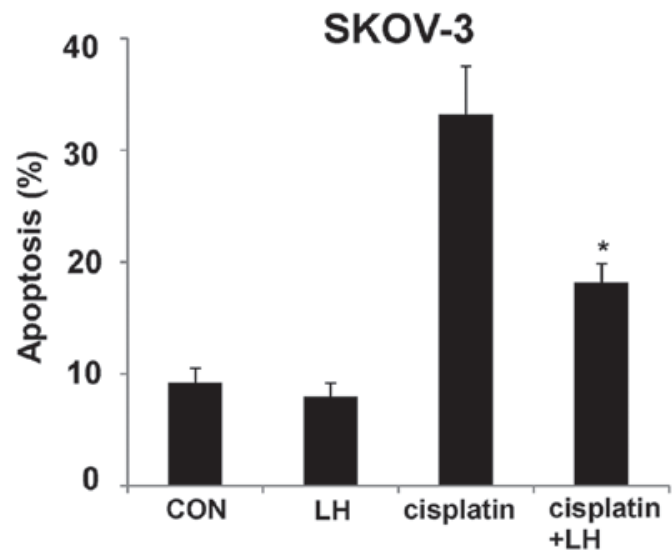

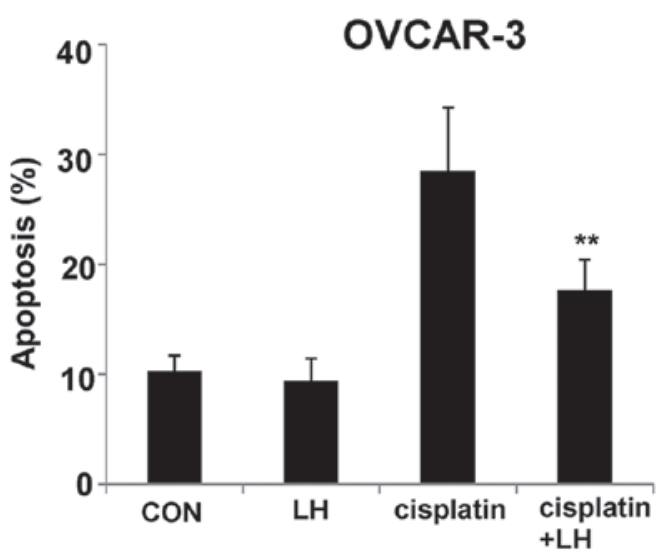

Figure 1. Effect of LH on ovarian cancer cell apoptosis. Apoptosis in the (A) SKOV-3 and (B) OVCAR-3 cells was measured using an apoptosis detection kit. ${ }^{*} \mathrm{P}<0.05$ vs. cisplatin-treated SKOV-3 cells and ${ }^{* *} \mathrm{P}<0.05$ vs. cisplatin-treated OVCAR-3 cells. LH, luteinizing hormone; PDCD6, programmed cell death gene 6; CON, control.

anti-human monoclonal antibody; cat. no. 3270; dilution, 1:1,000), c-Jun (rabbit anti-human monoclonal antibody; cat. no. 9165; dilution, 1:1,000), phospho-p38 MAPK (rabbit anti-human monoclonal antibody; cat. no. 4511; dilution, 1:1,000), p38 MAPK (rabbit anti-human monoclonal antibody; cat. no. 9212; dilution, 1:1,000), phospho-p44/42 MAPK (pErk1/2; rabbit anti-human monoclonal antibody; cat. no. 4370; dilution, 1:1,000), and extracellular signal-regulated protein kinase 1 and 2 (Erk1/2; rabbit anti-human monoclonal antibody; cat. no. 9102; dilution, 1:1,000) were purchased from Cell Signaling Technology, Inc. (Danvers, MA, USA). The glyceraldehyde 3-phosphate dehydrogenase (GAPDH) antibody (mouse anti-human monoclonal antibody; cat. no. KC-5G5; dilution, 1:10,000) was purchased from Kangchen Bioengineering Corporation (Shanghai, China).

Cell culture. The ovarian cancer cell lines, OVCAR-3 and SKOV-3, were purchased from the American Type Culture Collection (Manassas, VA, USA) and cultured according to the company's protocols. SKOV-3 cells were cultured at $37^{\circ} \mathrm{C}$ with $5 \% \mathrm{CO}_{2}$ in McCoy's 5a medium (Gibco; Thermo Fisher Scientific, Inc., Waltham, MA, USA) supplemented with $10 \%$ fetal bovine serum (FBS; Gibco; Thermo Fisher Scientific, Inc.) and $0.1 \%$ gentamicin sulfate (Gemini Bio Products, West Sacramento, CA, USA), while OVCAR-3 cells were cultured in RPMI-1640 medium (Gibco; Thermo Fisher Scientific, Inc.) supplemented with $10 \%$ FBS and $0.1 \%$ gentamicin sulfate. All experiments were performed with cell lines at $60 \%$ to $80 \%$ confluence. To investigate the expression of PDCD6, the cells were treated for up to $24 \mathrm{~h}$ with $\mathrm{LH}$ (40 U/l) in the absence of FBS. To investigate signal transduction, the cells were treated for up to $120 \mathrm{~min}$ with LH (40 U/l) in the absence of FBS. To evaluate the effects of the specific inhibitors, the cells were pre-treated with each inhibitor for $30 \mathrm{~min}$, and $\mathrm{LH}$ was then added for an additional $30 \mathrm{~min}$ or $24 \mathrm{~h}$ without FBS.

Cell apoptosis analysis. Following a 48-h incubation with LH, with or without cisplatin $(10 \mu \mathrm{M})$, the number of apoptotic cells was determined using the Annexin V-FITC Apoptosis Detection kit (BD Pharmingen, San Diego, CA, USA) followed by flow cytometry. The apoptotic cells were determined using a FACScan cytofluorometer from BD Biosciences (Franklin Lakes, NJ, USA) with Cell Quest software version 5.1, also from BD Biosciences. The early apoptotic [Annexin-V positive, propidium iodide (PI) negative] and late apoptotic (Annexin-V positive, PI positive) cells were included in cell death determinations.

$q P C R$. Total RNA was prepared using the RNAprep Pure Cell kit (Tiangen Biotech Co., Ltd., Beijing, China) according to the manufacturer's protocols. The primers utilized for the SYBR Green (Tiangen Biotech Co., Ltd.) qPCR were as follows: PDCD6, 5'-GGATGATCGATAAGAACGAGC TGAA-3' (forward) and 5'-ATGAGGATGTCGTGGAAC TGGTC-3' (reverse); and GAPDH, 5'-ATGGAAATCCCA TCACCATCTT-3' (forward) and 5'-CGCCCCACTTGATTT TGG-3' (reverse). The reaction mixture was composed of $12.5 \mu$ l One Step SYBR RT-PCR Buffer III, 0.5 $\mu 1$ Takara Ex Taq HS DNA Polymerase (5 U/ $\mu \mathrm{l}), 0.5 \mu \mathrm{l}$ PrimeScript RT Enzyme Mix II, $0.5 \mu \mathrm{l}$ Forward PCR Primer $(10 \mu \mathrm{M}), 0.5 \mu \mathrm{l}$ Reverse PCR Primer (10 $\mu \mathrm{M}), 2 \mu \mathrm{l}$ total RNA (100 ng) and $8.5 \mu \mathrm{l}$ RNase Free $\mathrm{dH}_{2} \mathrm{O}$ (Thermo Fisher Scientific, Inc.), all obtained from Takara Bio, Inc. (Otsu, Japan). The qPCR conditions in an Applied Biosystems 7500 series qPCR system (Thermo Fisher Scientific, Inc.) were as follows: $42^{\circ} \mathrm{C}$ for $5 \mathrm{~min}$, followed by $95^{\circ} \mathrm{C}$ for $10 \mathrm{sec}$, then 40 cycles of $95^{\circ} \mathrm{C}$ for $5 \mathrm{sec}$ and $60^{\circ} \mathrm{C}$ for $30 \mathrm{sec}$. RNase-Free $\mathrm{dH}_{2} \mathrm{O}$ without RNA was set as a negative control. The relative mRNA expression levels were calculated and normalized using the qPCR and the $2^{-\Delta \Delta \mathrm{cq}}$ method.

Western blotting. Following cell lysis, $30 \mu \mathrm{g}$ total protein was separated on $8-12 \%$ sodium dodecyl sulfate-polyacrylamide gels, transferred to nitrocellulose membranes (GE Healthcare Life Sciences, Uppsala, Sweden) and immunoblotted with specific primary antibodies (PDCD6, GAPDH, pAKT, AKT, pJUN,c-Jun,phospho-p44/42MAPK,p38MAPK,phospho-p38 MAPK, pErk1/2, and Erk1/2) at $4^{\circ} \mathrm{C}$ overnight. Subsequently, secondary antibody (sheep anti-rabbit IgG secondary antibody; cat. no. KC-RB-035; dilution, 1:5,000; or sheep anti-mouse IgG secondary antibody; cat no. KC-MM-1302; dilution, 1:1,000; Kangchen Bioengineering Co.) incubations were performed 
A

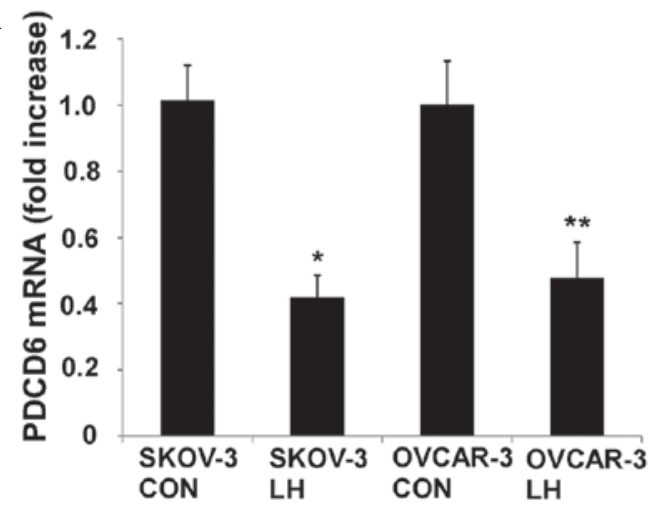

B

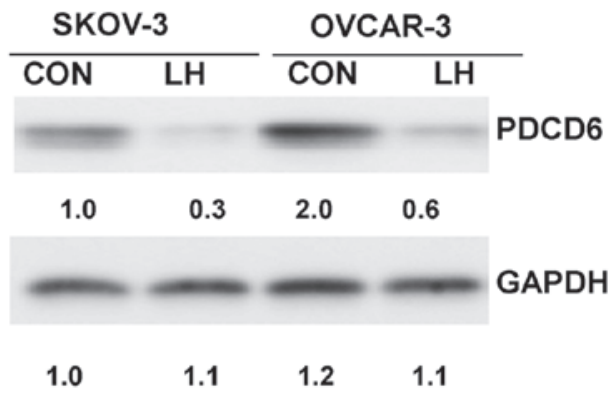

Figure 2. PDCD6 expression induced by LH. (A) PDCD6 mRNA was measured by quantitative polymerase chain reaction and (B) PDCD6 protein was measured by western blotting. " $\mathrm{P}<0.05$ vs. control SKOV-3 cells and ${ }^{* *} \mathrm{P}<0.05$ vs. control OVCAR-3 cells. PDCD6, programmed cell death gene 6; LH, luteinizing hormone; CON, control; GAPDH, glyceraldehyde 3-phosphate dehydrogenase.
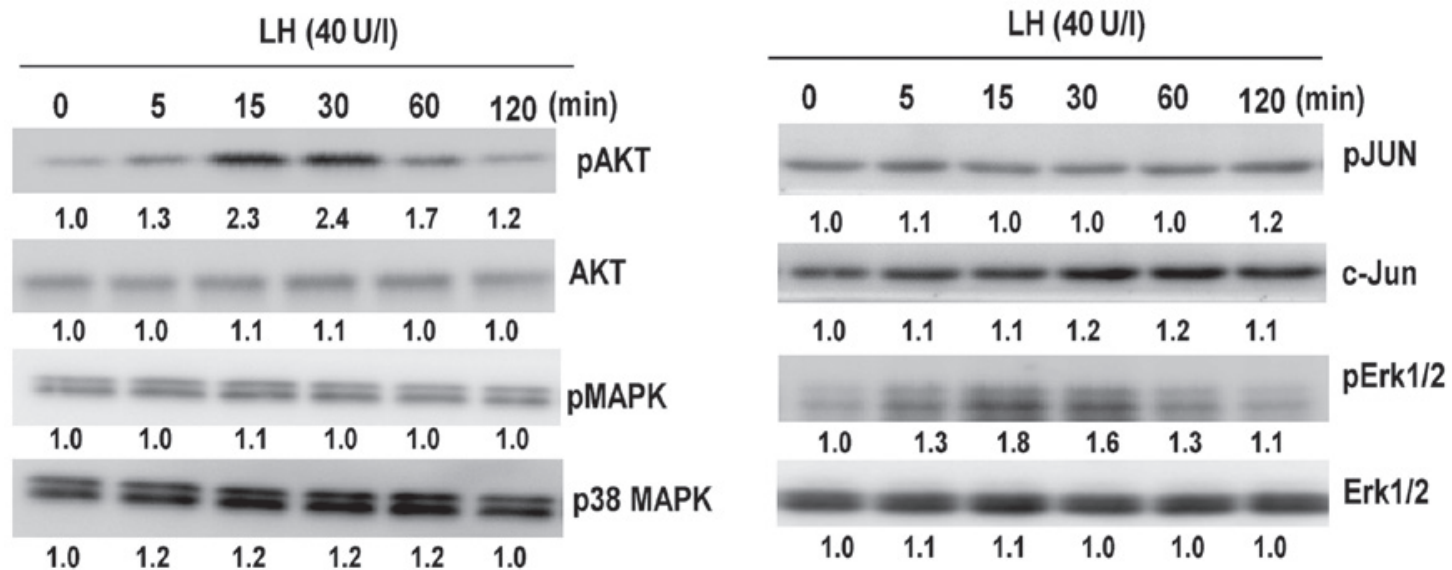

Figure 3. Western blot analysis demonstrating the signal transduction pathways induced by LH. Western blot analysis was performed following treatment with $40 \mathrm{U} / 1 \mathrm{LH}$ for up to $120 \mathrm{~min}$ in the SKOV-3 cells. LH, luteinizing hormone; pAKT, phospho-AKT; MAPK, mitogen-activated protein kinase; pMAPK, phospho-p38 MAPK; pJUN, phosphorylated c-Jun; Erk1/2, extracellular signal-regulated kinase 1/2; pErk1/2, phospho-Erk1/2.
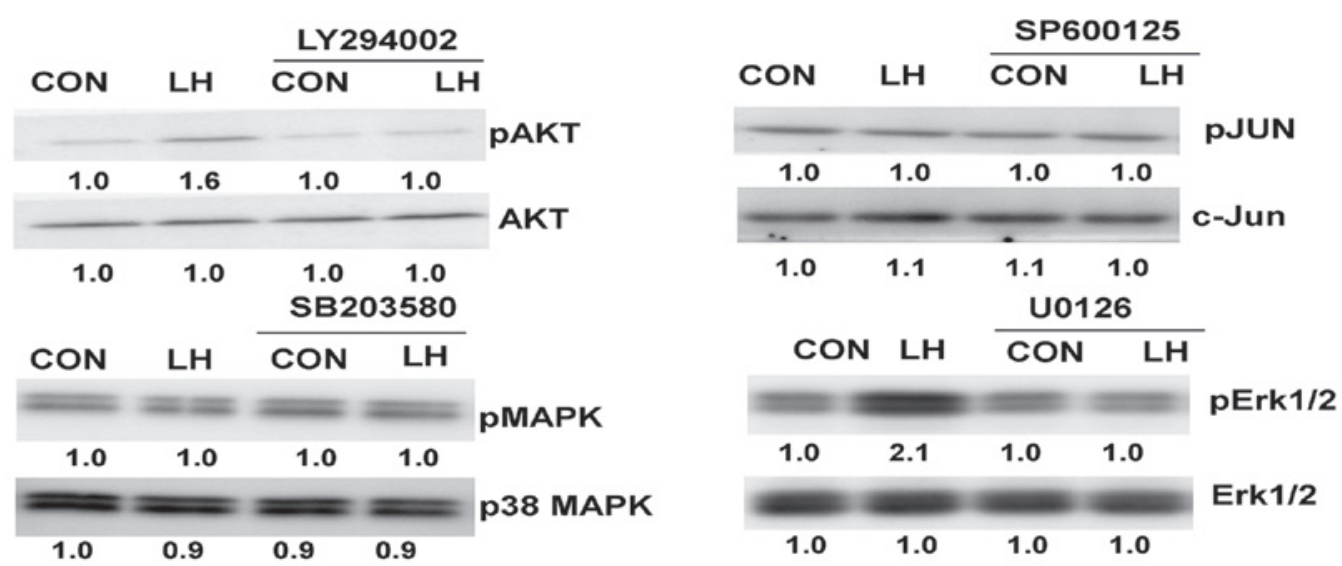

Figure 4. Western blot analysis demonstrating suppression of phosphorylation by the inhibitors. Following a 30-min pre-treatment with $10 \mu \mathrm{M}$ LY294002 (PI3K inhibitor), $10 \mu \mathrm{M}$ SP600125 (SAPK/JNK inhibitor), $10 \mu \mathrm{M}$ U0126 (Erk1/2 inhibitor) or $20 \mu \mathrm{M}$ SB203580 (p38 MAPK inhibitor), the cells were treated with $40 \mathrm{U} / 1 \mathrm{LH}$ for an additional $30 \mathrm{~min}$. LH, luteinizing hormone; pAKT, phospho-AKT; MAPK, mitogen-activated protein kinase; pMAPK, phospho-p38 MAPK; pJUN, phosphorylated c-Jun; Erk1/2, extracellular signal-regulated kinase 1/2; pErk1/2, phospho-Erk1/2; CON, control.

at room temperature for $60 \mathrm{~min}$. The signals were detected using the Amersham ECL Western Blotting Detection kit (GE Healthcare Life Sciences, Uppsala, Sweden). GAPDH was used for the loading control. Densitometry (Image J; National Institutes of Health, Bethesda, MD, USA) was used to assess the differences in the results. 

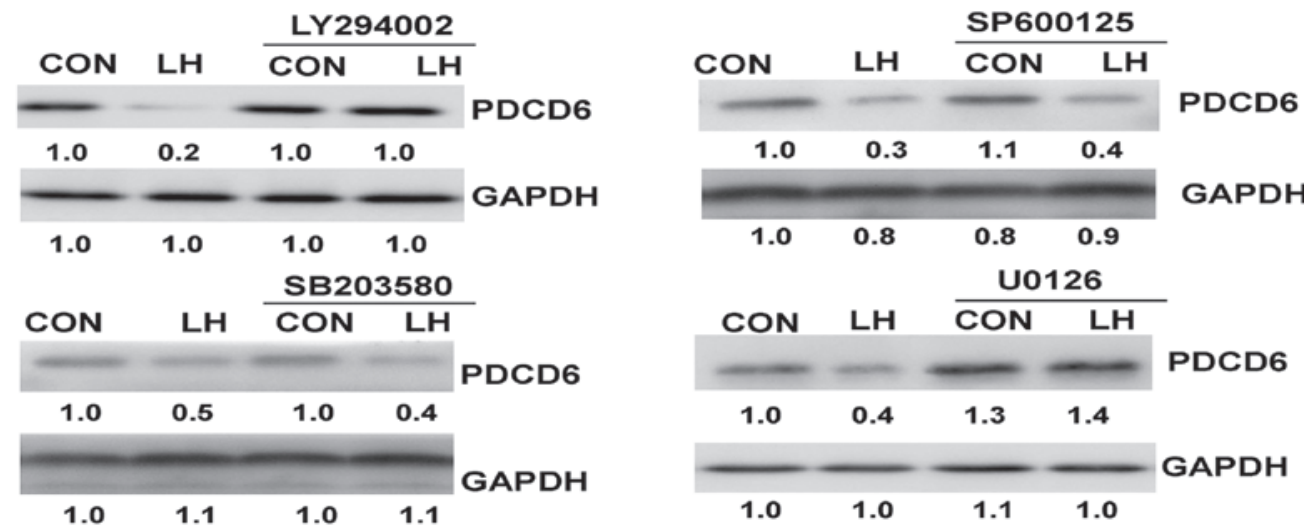

Figure 5. Western blot analysis demonstrating that the LH-induced PDCD6 protein is neutralized by the inhibitors. Following a 30-min pre-treatment with $10 \mu \mathrm{M}$ LY294002 (PI3K inhibitor), $10 \mu \mathrm{M}$ SP600125 (SAPK/JNK inhibitor), $10 \mu \mathrm{M}$ U0126 (Erk1/2 inhibitor) or $20 \mu \mathrm{M}$ SB203580 (p38 MAPK inhibitor), the cells were treated with $40 \mathrm{U} / 1 \mathrm{LH}$ for an additional $24 \mathrm{~h}$. LH, luteinizing hormone; CON, control; GADPH, glyceraldehyde 3-phosphate dehydrogenase; PDCD6, programmed cell death gene 6.

Statistical analysis. The data averages were based on three individual experiments that were performed in triplicate. Results for the experiments were analyzed using Student's t-test. $\mathrm{P}<0.05$ was considered to indicate a statistically significant difference. SPSS software, version 11.0 (SPSS, Inc., Chicago, IL, USA), was used for all statistical analyses.

\section{Results}

Effect of LH on ovarian cancer cell apoptosis. Due to the prevalence of ovarian cancer in post-menopausal women with $\sim 40 \mathrm{U} / 1 \mathrm{LH}$, this concentration was chosen for the present study (8). As presented in Fig. 1, LH treatment resulted in a slight decrease in the rate of apoptosis when compared with controls in the SKOV-3 and OVCAR-3 cells, however, the difference was not significant $(\mathrm{P}>0.05)$. To determine whether LH could block apoptosis induced by cisplatin, the SKOV-3 and OVCAR-3 cells were treated with $10 \mu \mathrm{M}$ cisplatin, or a combination of $40 \mathrm{U} / 1 \mathrm{LH}$ and $10 \mu \mathrm{M}$ cisplatin. As presented in Fig. 1, the rate of apoptosis induced by cisplatin was significantly suppressed by LH $(\mathrm{P}<0.05)$.

PDCD6 expression is inhibited by LH. The SKOV-3 and OVCAR-3 cells were treated with $40 \mathrm{U} / 1 \mathrm{LH}$ for up to $24 \mathrm{~h}$. qPCR indicated that PDCD6 mRNA was significantly downregulated when treated with $\mathrm{LH}$ for $16 \mathrm{~h}$ ( $\mathrm{P}<0.05$; Fig. 2A). Additionally, western blotting analysis demonstrated that the protein expression of PDCD6 decreased when cells were treated with LH for $24 \mathrm{~h}(\mathrm{P}<0.05)$ (Fig. 2B).

Signaling transduction pathways induced by $L H$. The present study investigated whether the SAPK/JNK, p38 MAPK, $\mathrm{PI} 3 \mathrm{~K} / \mathrm{AKT}$ and Erk1/2 signaling pathways are involved in LH-mediated apoptosis. Due to LH exerting similar effects on each of the cell lines, signaling was only analyzed in the SKOV-3 cells. It was observed that LH induced the phosphorylation of AKT and Erk1/2 ( $\mathrm{P}<0.05$; Fig. 3), however, it did not affect the phosphorylation of p38 MAPK and c-Jun ( $\mathrm{P}>0.05$.

LH-induced phosphorylation is blocked by inhibitors. The SKOV-3 and OVCAR-3 cells were pre-treated with the inhibitors for $30 \mathrm{~min}$, and LH was then added for an additional 30 min. A pre-treatment with LY294002 or U0126 blocked LH-induced phosphorylation (Fig. 4).

The LH-induced PDCD6 protein inhibition is neutralized by the inhibitors. The SKOV-3 and OVCAR-3 cells were pre-treated with the inhibitors for $30 \mathrm{~min}$, and LH was then added for an additional 24 h. LY294002 and U0126 significantly neutralized LH-induced PDCD6 protein inhibition $(\mathrm{P}<0.05$; Fig. 5), however, SP600125 and SB203580 did not have the same effect $(\mathrm{P}>0.05)$.

\section{Discussion}

The majority of ovarian tumors develop in post-menopausal women presenting with high gonadotropin levels. Thus, gonadotropins are regarded as possible risk factors for the formation of ovarian tumors (2). In 1992, Ohtani et al (9) observed that FSH stimulated proliferation in ovarian cancer cells; subsequent to this, it was reported by Zheng et al (10) that ovarian epithelial tumor growth was promoted by FSH and inhibited by LH. Choi et al (11) demonstrated that gonadotropins upregulated epidermal growth factor receptors (EGFRs) through the activation of MAPK and PI3K in human ovarian surface epithelial cells. In a further study by Choi et al (12), it was observed that gonadotropins activated proteolysis and increased invasion through the protein kinase A (PKA) and PI3K pathways in human epithelial ovarian cancer cells. Apoptosis serves an important role in the progression of ovarian cancer (13), and certain papers have suggested that human chorionic gonadotropin or LH may inhibit cisplatin-induced apoptosis in ovarian cancer cells $(14,15)$.

PDCD6 is an important apoptotic mediator and a prognostic marker for gastric cancer (16); it is also an independent predictor of progression-free survival in patients with epithelial ovarian cancer (7). Park et al (17) demonstrated that PDCD6 additively cooperated with anticancer drugs through the activation of nuclear factor- $\kappa \mathrm{B}$ pathways. It was previously reported that FSH decreased PDCD6 levels and inhibited the rate of apoptosis in ovarian cancer cells (5). In the present study, it was observed that LH significantly blocked 
cisplatin-induced apoptosis in vitro. Furthermore, LH significantly reduced PDCD6 expression. Such results suggest that the downregulation of PDCD6, induced by LH, appears to be involved in the chemoresistance of ovarian cancer due to the induction of apoptosis inhibition.

Numerous studies have focused on the signaling pathways induced by gonadotropins in ovarian cancer cells and in human ovarian surface epithelium (OSE). Pon et al (18) reported that gonadotropins reduced $\mathrm{N}$-cadherin expression in OSE cells through the PKA pathway. It has also been demonstrated that EGFR expression is regulated by gonadotropins via cyclic adenosine monophosphate, and this does not signal through PKA in immortalized OSE cells (19). FSH-induced DNA synthesis and proliferation may be neutralized by a number of protein kinase $\mathrm{C}$ (PKC) inhibitors, implying that the $\mathrm{PKC}$ pathway is also associated with FSH-induced cell growth (20). It was previously reported that FSH induced vascular endothelial growth factor through the PI3K/AKT pathway (21). Slot et al (22) first demonstrated that LH protected HEY cells from Fas-induced apoptosis, occurring via a signaling cascade involving PKA. The Erk1/2 pathway has been identified to be involved in LH-induced survivin expression and apoptosis (15). A previous study indicated that FSH induced PDCD6 expression through activation of the PI3K/AKT and SAPK/JNK signaling pathways, and that treatment with LY294002, the specific PI3K inhibitor, antagonized the effects of FSH on pAKT and PDCD6 expression; furthermore, the study reported that treatment with SP600125, the specific SAPK/JNK inhibitor, antagonized the effects of FSH on pJUN and PDCD6 expression (5). It remains unknown whether FSH and $\mathrm{LH}$ regulate PDCD6 expression in ovarian cancer through different or identical signaling pathways. The results of the present study indicate that LH inhibits PDCD6 expression in ovarian cancer cells by activating the PI3K/AKT and Erk1/2 signaling pathways.

In conclusion, the current study demonstrated that $\mathrm{LH}$ inhibited cisplatin-induced apoptosis and suppressed PDCD6 in ovarian cancer cells. LH inhibited PDCD6 through the activation of the PI3K/AKT and Erk1/2 signaling pathways. The results suggest that LH serves a critical role in the chemoresistance of ovarian cancer based on the anti-apoptotic effect it induces.

\section{References}

1. Siegel R, Ma J, Zou Z and Jemal A: Cancer statistics, 2014. CA Cancer J Clin 64: 9-29, 2014.

2. Mertens-Walker I, Baxter RC and Marsh DJ: Gonadotropin signalling in epithelial ovarian cancer. Cancer Lett 324: 152-159, 2012.

3. Dong Z, Yang L and Lai D: KLF5 strengthens drug resistance of ovarian cancer stem-like cells by regulating survivin expression. Cell Prolif 46: 425-435, 2013.

4. Duiker EW, Meijer A, van der Bilt AR, Meersma GJ, Kooi N, van der Zee AGJ, de Vries EG and de Jong S: Drug-induced caspase 8 upregulation sensitises cisplatin-resistant ovarian carcinoma cells to rhTRAIL-induced apoptosis. Br J Cancer 104: 1278-1287, 2011.

5. Huang Y, Jin H, Liu Y, Zhou J, Ding J, Cheng KW, Yu Y and Feng Y: FSH inhibits ovarian cancer cell apoptosis by up-regulating survivin and down-regulating PDCD6 and DR5. Endocr Relat Cancer 18: 13-26, 2011.
6. Vito $\mathrm{P}$, Lacanà $\mathrm{E}$ and D'Adamio L: Interfering with apoptosis: $\mathrm{Ca}(2+)$-binding protein ALG-2 and Alzheimer's disease gene ALG-3. Science 271: 521-525, 1996.

7. Su D, Xu H, Feng J, Gao Y, Gu L, Ying L, Katsaros D, Yu H, Xu S and Qi M: PDCD6 is an independent predictor of progression free survival in epithelial ovarian cancer. J Transl Med 10: 31, 2012.

8. Brodowska A, Laszczyńska M, Brodowski J, Masiuk M and Starczewski A: Analysis of pituitary gonadotropin concentration in blood serum and immunolocalization and immunoexpression of follicle stimulating hormone and luteinising hormone receptors in ovaries of postmenopausal women. Histol Histopathol 27: 241-248, 2012.

9. Ohtani K, Sakamoto H and Satoh K: Stimulatory effects of follicular stimulating hormone on the proliferation of ovarian cancer cell line in vitro and in vivo. Nihon Sanka Fujinka Gakkai Zasshi 44: 717-724, 1992 (In Japanese).

10. Zheng W, Lu JJ, Luo F, Zheng Y, Feng Y, Felix JC, Lauchlan SC and Pike MC: Ovarian epithelial tumor growth promotion by follicle-stimulating hormone and inhibition of the effect by luteinizing hormone. Gynecol Oncol 76: 80-88, 2000.

11. Choi JH, Choi KC, Auersperg N and Leung PC: Gonadotropins upregulate the epidermal growth factor receptor through activation of mitogen-activated protein kinases and phosphatidyl-inositol-3-kinase in human ovarian surface epithelial cells. Endocr Relat Cancer 12: 407-421, 2005.

12. Choi JH, Choi KC, Auersperg N and Leung PC: Gonadotropins activate proteolysis and increase invasion through protein kinase A and phosphatidylinositol 3-kinase pathways in human epithelial ovarian cancer cells. Cancer Res 66: 3912-3920, 2006.

13. Zhao JX, Liu H, Lv J and Yang XJ: Wortmannin enhances cisplatin-induced apoptosis in human ovarian cancer cells in vitro. Eur Rev Med Pharmacol Sci 18: 2428-2434, 2014.

14. Kuroda H, Mandai M, Konishi I, Yura Y, Tsuruta Y, Hamid AA, Nanbu K, Matsushita K and Mori T: Human chorionic gonadotropin (hCG) inhibits cisplatin-induced apoptosis in ovarian cancer cells: possible role of up-regulation of insulin-like growth factor-1 by hCG. Int J Cancer 76: 571-578, 1998.

15. Zhang Z, Liao H, Chen X, Zheng Y, Liu Y, Tao X, Gu C, Dong L, Duan T, Yang Y, et al: Luteinizing hormone upregulates survivin and inhibits apoptosis in ovarian epithelial tumors. Eur J Obstet Gynecol Reprod Biol 155: 69-74, 2011.

16. Yoon JH, Choi YJ, Kim SG, Nam SW, Lee JY and Park WS: Programmed cell death 6 (PDCD6) as a prognostic marker for gastric cancers. Tumour Biol 33: 485-494, 2012.

17. Park SH, Lee JH, Lee GB, Byun HJ, Kim BR, Park CY, Kim HB and Rho SB: PDCD6 additively cooperates with anti-cancer drugs through activation of NF- $\kappa$ B pathways. Cell Signal 24: 726-733, 2012.

18. Pon YL, Auersperg N and Wong AS: Gonadotropins regulate $\mathrm{N}$-cadherin-mediated human ovarian surface epithelial cell survival at both post-translational and transcriptional levels through a cyclic AMP/protein kinase A pathway. J Biol Chem 280: 15438-15448, 2005.

19. Choi JH, Chen CL, Poon SL, Wang HS and Leung PC: Gonadotropin-stimulated epidermal growth factor receptor expression in human ovarian surface epithelial cells: Involvement of cyclic AMP-dependent exchange protein activated by cAMP pathway. Endocr Relat Cancer 16: 179-188, 2009.

20. Ohtani K, Sakamoto H, Kikuchi A, Nakayama Y, Idei T, Igarashi N, Matukawa T and Satoh K: Follicle-stimulating hormone promotes the growth of human epithelial ovarian cancer cells through the protein kinase $\mathrm{C}$-mediated system. Cancer Lett 166: 207-213, 2001.

21. Huang Y, Hua K, Zhou X, Jin H, Chen X, Lu X, Yu Y, Zha X and Feng Y: Activation of the PI3K/AKT pathway mediates FSH-stimulated VEGF expression in ovarian serous cystadenocarcinoma. Cell Res 18: 780-791, 2008.

22. Slot KA, de Boer-Brouwer M, Houweling M, Vaandrager AB, Dorrington JH and Teerds KJ: Luteinizing hormone inhibits Fas-induced apoptosis in ovarian surface epithelial cell lines. J Endocrinol 188: 227-239, 2006. 